\title{
Análise dos casos de gripe A(H1N1) no Brasil e no estado do Maranhão de 2009 a 2019
}

Analysis of cases of influenza A(H1N1) in Brazil and the state of Maranhão from 2009 to 2019

Análisis de casos de influenza A(H1N1) en Brasil y el estado de Maranhão de 2009 a 2019

\author{
Alejandro Elias Mouchrek Jaldin \\ ORCID: https://orcid.org/0000-0002-6347-2166 \\ Universidade Federal do Maranhão, Brasil \\ E-mail: alejandro.jaldin@hotmail.com \\ Luana Mendes Nogueira \\ ORCID: https://orcid.org/0000-0002-6790-0674 \\ Universidade Federal do Maranhão, Brasil \\ E-mail: luanamendesnogueira@gmail.com \\ Nilson de Jesus Pereira Batalha Junior \\ ORCID: https://orcid.org/0000-0002-6325-5655 \\ Universidade Federal do Maranhão, Brasil \\ E-mail: nilsonbatalha@gmail.com \\ Consuelo Penha Castro Marques \\ ORCID: https://orcid.org/0000-0002-2149-5300 \\ Universidade Federal do Maranhão, Brasil \\ E-mail: consuelo.penha@ufma.br \\ Izolda Souza Costa \\ ORCID: https://orcid.org/0000-0003-0899-6845 \\ Universidade Federal do Maranhão, Brasil \\ E-mail: izolda.costa@hotmail.com \\ Jefferson Rummenigge Nascimento Campos \\ ORCID: https://orcid.org/0000-0003-4251-8169 \\ Universidade Federal do Maranhão, Brasil \\ E-mail: jeffersonrnc@gmail.com \\ Wilken Soares Batista \\ ORCID: https://orcid.org/0000-0002-6264-1167 \\ Universidade Federal do Maranhão, Brasil \\ E-mail: wilkennsb@hotmail.com \\ Daniel Santos Uchoa \\ ORCID: https://orcid.org/0000-0003-0456-4471 \\ Universidade Federal do Maranhão, Brasil \\ E-mail: danieluchoa95@gmail.com \\ Sueli de Souza Costa \\ ORCID: https://orcid.org/0000-0003-4127-7324 \\ Universidade Federal do Maranhão, Brasil \\ E-mail: sueli.costa@ufma.br
}

\begin{abstract}
Resumo
Desde meados de 2009, o Brasil convive com o vírus influenza A(H1N1)pdm09. No período pandêmico, o país notificou 50.482 casos de Síndrome Respiratória Aguda Grave (SRAG) por influenza A(H1N1)pdm09, dentre os quais 2.060 evoluíram com óbito. O objetivo deste estudo foi realizar uma análise de casos e óbitos notificados da gripe H1N1 no Brasil e no estado do Maranhão, de 2009 a 2019. Trata-se de um estudo ecológico sobre a gripe influenza $\mathrm{A}(\mathrm{H} 1 \mathrm{N1})$ pdm09. Os dados foram obtidos no Sistema de Informação de Agravos de Notificação (SINAN), do Ministério da Saúde (MS), disponível no banco de dados do Departamento de Informática do Sistema Único de Saúde - DATASUS. Confirmou-se a sazonalidade do vírus influenza A(H1N1)pdm09, ressalvadas as peculiaridades de cada região, principalmente no Norte e Centro Oeste do país. Os grupos de risco de destaque foram os adultos $>60$ anos, cardiopatas, pneumopatas e portadores de diabetes mellitus. O Maranhão registrou números abaixos da média nacional, sugerindo subnotificação. Os anos de 2016, 2018 e 2019 registraram os maiores números de casos e mortes, excluído o ano da pandemia, em 2019 (10.625/1.987, 3.880/917 e 3.399/787, respectivamente). Os estados do Sul e Sudeste apresentaram as maiores incidências em todo o período, com destaque para o Paraná e São Paulo. Diante dos resultados, notou-se a necessidade de reforço do sistema de vigilância sanitária da influenza, através da criação de mais unidades sentinelas e rigoroso controle dos dados; bem como otimização das campanhas de vacinação, visando se adequar à realidade encontrada em cada extremo do país.
\end{abstract}

Palavras-chave: Vírus; Influenza; Epidemiologia; Saúde pública. 


\begin{abstract}
Since mid-2009, Brazil has been living with the influenza A (H1N1) pdm09 virus. In the pandemic period, the country reported 50,482 cases of Severe Acute Respiratory Syndrome (SARS) due to influenza A (H1N1) pdm09, among which 2,060 died. The objective of this study was to carry out an analysis of notified cases and deaths of the H1N1 flu in Brazil and in the State of Maranhão, from 2009 to 2019. This is an ecological study on influenza A (H1N1) pdm09. The data were obtained from the Notifiable Diseases Information System (SINAN), of the Ministry of Health (MS), available in the database of the Informatics Department of the Unified Health System - DATASUS. The seasonality of influenza A (H1N1) pdm09 was confirmed, except for the peculiarities of each region, mainly in the North and Midwest of the country. The prominent risk groups were adults $>60$ years of age, heart disease, pulmonary disease and diabetes mellitus. Maranhão registered lower numbers than the national average, suggesting underreporting. The years 2016, 2018 and 2019 recorded the highest number of cases and deaths, excluding the year of the pandemic, in 2019 (10,625 / 1,987, 3,880 / 917 and 3,399 / 787, respectively). The states of the South and Southeast had the highest incidence in the entire period, with emphasis on Paraná and São Paulo. In view of the results, there was a need to reinforce the health surveillance system for influenza, through the creation of more sentinel units and strict data control; as well as optimization of vaccination campaigns, aiming to adapt to the reality found in each extreme of the country.
\end{abstract}

Keywords: Virus; Influenza; Epidemiology; Public health.

\title{
Resumen
}

Desde mediados de 2009, Brasil vive con el virus de influenza A (H1N1) pdm09. En el período pandémico, el país notificó 50.482 casos de Síndrome Respiratorio Agudo Severo (SARS) por influenza A (H1N1) pdm09, de los cuales 2.060 fallecieron. El objetivo de este estudio fue realizar un análisis de casos notificados y muertes por influenza H1N1 en Brasil y en el estado de Maranhão, de 2009 a 2019. Se trata de un estudio ecológico sobre influenza A (H1N1) pdm09. Los datos se obtuvieron del Sistema de Información de Enfermedades Notificables (SINAN), del Ministerio de Salud (MS), disponible en la base de datos del Departamento de Informática del Sistema Único de Salud - DATASUS. Se confirmó la estacionalidad de la influenza A (H1N1) pdm09, excepto por las peculiaridades de cada región, principalmente en el Norte y Medio Oeste del país. Los grupos de riesgo destacados fueron los adultos> 60 años, las enfermedades cardíacas, las enfermedades pulmonares y la diabetes mellitus. Maranhão registró cifras por debajo de la media nacional, lo que sugiere que no se informa. Los años 2016, 2018 y 2019 registraron el mayor número de casos y muertes, excluyendo el año de la pandemia, en 2019 (10,625 / 1,987, 3,880 / 917 y 3,399 / 787, respectivamente). Los estados del Sur y Sudeste tuvieron la mayor incidencia en todo el período, con énfasis en Paraná y São Paulo. A la vista de los resultados, era necesario fortalecer el sistema de vigilancia en salud de influenza, mediante la creación de más unidades centinela y un estricto control de datos; así como la optimización de las campañas de vacunación, con el objetivo de adaptarse a la realidad que se encuentra en cada extremo del país.

Palabras clave: Virus; Influenza; Epidemiología; Salud pública.

\section{Introdução}

No dia 11 de junho de 2009, a Organização Mundial de Saúde (OMS) decretou a pandemia do novo vírus da gripe classificado à época como A/California/04/09, sendo posteriormente chamado de influenza A(H1N1)pdm09, como ficou conhecido até os dias de hoje. Os primeiros casos da doença surgiram no México e, devido às suas origens em suínos, rapidamente também foi denominada como "gripe suína” (Lobo, 2015).

A nova ameaça biológica mundial circulou por cerca de 214 países e "até agosto de 2010, mês no qual a OMS anunciou a transição do período pandêmico para o pós-pandêmico, foram registradas mais de 18.449 mortes" (Felinto, Escosteguy, \& Medronho, 2018). No Brasil, em 2009, antes mesmo de completar um ano da presença do vírus no país, foram notificados 88.464 casos de Síndrome Respiratória Aguda Grave (SRAG), sendo 50.482 confirmados para influenza $\mathrm{A}(\mathrm{H} 1 \mathrm{N1}) \mathrm{pdm} 09$, dentre os quais 2.060 evoluíram com óbito (Brasil, 2012).

Cerca de dez anos após o surgimento da gripe H1N1, uma nova doença passou a afligir o mundo todo. A partir dos primeiros relatos e estudos, "pacientes com pneumonia de causa desconhecida, epidemiologicamente ligados a um mercado atacadista de frutos do mar em Wuhan-China”, foram identificados com uma nova espécie do gênero betacoronavírus, o qual poderia ser responsável por uma Síndrome Respiratória Aguda Grave (SRAG) análoga a outra anteriormente já identificada em pessoas infectadas por outra espécie desse mesmo vírus (Zhu et al., 2020).

Semelhantemente ao que ocorreu em 2009, a doença provocada pelo coronavírus também adquiriu o status de 
pandemia pela OMS em 11 de março de 2020 (WHO, 2020). A nova moléstia apresenta características bem similares ao da gripe H1N1, como os sintomas, forma de transmissão e prevenção, grupos de risco (idosos), entre outros (Tang et al., 2020). Tal fator foi preponderante no enfrentamento à pandemia mais recente, levando o Ministério da Saúde (MS) do Brasil a adotar, como uma de suas estratégias, o adiantamento da campanha de vacinação contra a influenza (Brasil, 2020a).

Dentro desse contexto, o governo do estado do Maranhão, por meio do Decreto Estadual no 35.672 , de 19 de março de 2020, declarou estado de calamidade pública "considerando o aumento brusco, significativo e transitório da ocorrência de doenças infecciosas geradas pelo vírus H1N1, bem como a existência de casos suspeitos de contaminação pela COVID-19" (Estado do Maranhão, 2020).

A identificação do aumento inusitado de casos da gripe H1N1 no estado do Maranhão, no início do ano de 2020, abriu o alerta das autoridades de saúde e também da população, os quais não esperavam essa realidade frente à já existência da vacina e outras formas de combate implementadas. No entanto, Coimbra (2010) já destacava a possibilidade de mudança no vírus e que "nos casos de epidemias, geralmente as ondas posteriores são mais fortes". Além disso, "não podemos minimizar os 'velhos vírus', que continuam causando problemas reais" e é natural que as pessoas fiquem com medo do coronavírus, e que a novidade cause impacto na população, "mas outras doenças como sarampo e gripe, que matam" deveriam ter sua devida atenção (O Globo, 2020).

Diante disso, entendendo que as doenças infecciosas, principalmente aquelas respiratórias provocadas por vírus, podem de tempos em tempos isoladamente - ou somado a ausência/relaxamento de políticas públicas voltadas à prevenção ocasionar surtos inadvertidamente; torna-se bastante relevante a necessidade de se fazer uma análise quanto ao número de casos notificados da gripe H1N1 no Brasil e no estado do Maranhão entre os anos de 2009 e 2020, a fim de identificar os motivos pelos quais o aumento ou diminuição dos casos têm ocorrido e, assim, subsidiar políticas públicas na área da saúde e o desenvolvimento de qualquer outro estudo vindouro a respeito.

\section{Metodologia}

Trata-se de um estudo ecológico sobre o número de casos e óbitos da gripe influenza A(H1N1)pdm09 no Brasil e no estado do Maranhão (Estrela, 2018). Estudos ecológicos comparam "a ocorrência da doença/condição relacionada à saúde e a exposição de interesse entre agregados de indivíduos (populações de países, regiões ou municípios, por exemplo)”, sendo que uma de suas vantagens é o fato de poder "examinar associações entre exposição e doença/condição relacionada na coletividade" (Lima-Costa \& Barreto, 2003).

A pesquisa foi realizada entre os anos de 2009 e 2019, por meio do Sistema de Informação de Agravos de Notificação (SINAN), do Ministério da Saúde (MS), disponível no banco de dados de domínio público do Departamento de Informática do Sistema Único de Saúde (DATASUS). O SINAN é responsável pelo funcionamento do sistema de vigilância epidemiológica de doenças transmissíveis. Seu objetivo é detectar com agilidade o surgimento de surtos e epidemias para que os órgãos públicos de saúde possam agir sobre ela o mais breve possível (Silva Junior et al., 2016).

A partir dos dados gerais coletados em cada ano, foi feito um filtro com as informações mais relevantes para análise e, em seguida, uma tabulação das informações por meio do programa Microsoft Excel® 2016. As variáveis utilizadas no estudo foram os números de casos e de mortes por gripe $\mathrm{A}(\mathrm{H} 1 \mathrm{~N} 1) \mathrm{pdm} 09$, considerando a região/estado, ano/mês de ocorrência; bem como os fatores de risco que influenciaram na evolução para o óbito.

Por se tratar de coleta de dados em plataforma digital aberta, este projeto ficou dispensado de apresentação a Comitê de Ética em Pesquisa, de acordo com as Resoluções 466/2012 e 580/2018 do Conselho Nacional de Saúde, do Ministério da Saúde. 


\section{Resultados e Discussão}

No Brasil, considerando a Constituição da República, que prevê a saúde como direito social básico de todas as pessoas e dever do Estado (artigos $6^{\circ}$ e 196 em diante), e a lei 8080/90, que em seu artigo $2^{\circ}$, parágrafo $1^{\circ}$ esclarece ser dever do Estado a garantia da saúde, devendo, para tal, formular e executar políticas econômicas e sociais "que visem à redução de riscos de doenças e de outros agravos e no estabelecimento de condições que assegurem acesso universal e igualitário às ações e aos serviços para a sua promoção, proteção e recuperação", a partir do ano 2000 foi implantado um Sistema de Vigilância Sentinela de Influenza, composto por unidades sentinelas localizadas em vários pontos do país, principalmente, nas capitais (Brasil, 1988; Brasil, 1990).

As unidades são responsáveis por alimentar o Sistema de Informação de Vigilância Epidemiológica da Influenza (SIVEP-Gripe) com os dados coletados semanalmente (Opendatasus, 2021). A partir desses dados, são publicados boletins epidemiológicos semanais, tendo como objetivo identificar os vírus respiratórios que circulam pelo país, além de permitir o monitoramento da demanda do atendimento dos casos hospitalizados e óbitos para orientar na tomada de decisão em situações que requeiram novos posicionamentos do Ministério da Saúde e Secretarias de Saúde Estaduais e Municipais.

A vigilância da influenza no Brasil é composta pela vigilância sentinela de Síndrome Gripal (SG), de Síndrome Respiratória Aguda Grave (SRAG) em pacientes internados em Unidade de Terapia Intensiva (UTI) e pela vigilância universal de SRGA. Em se tratando da SG, o monitoramento ocorre por meio da coleta, pelas unidades sentinelas, de 05 (cinco) amostras de secreção da nasofaringe por semana; através das quais é feita a pesquisa para os vírus influenza, parainfluenza, adenovírus e vírus sincicial respiratório (VSR). Por meio desse método, tem-se apenas uma projeção dos principais vírus que circulam no país e em que período do ano mais se apresentam (Opendatasus, 2021).

Através dos dados coletados, observou-se que, excluídos os anos de 2010, 2014, 2015 e 2017, os demais anos entre 2009 e 2019 apresentaram um certo padrão quanto ao período de crescimento/pico/diminuição de ocorrência do vírus influenza A(H1N1)pdm09. O período de maior circulação desse vírus ocorreu entre a $10^{\mathrm{a}}$ e $40^{\mathrm{a}}$ semana epidemiológica (SE) e os gráficos nesses anos apresentaram uma curva bem similar, mostrando: um número relativamente baixo de casos no início do ano, aumento gradativo por volta da $10^{\mathrm{a}}$ semana, atingindo o seu pico, e redução gradativa até a estabilização de um número baixo de casos, encerrando o ano (Tabela 1).

Tabela 1. Aumento gradativo, pico e redução com estabilização dos casos de Influenza A(H1N1).

\begin{tabular}{c|c|c|c|c|c|c|c}
\cline { 2 - 8 } & $\mathbf{2 0 0 9}$ & $\mathbf{2 0 1 1}$ & $\mathbf{2 0 1 2}$ & $\mathbf{2 0 1 3}$ & $\mathbf{2 0 1 6}$ & $\mathbf{2 0 1 8}$ & $\mathbf{2 0 1 9}$ \\
\hline Início do aumento gradativo & $11^{\mathrm{a}}$ & $10^{\mathrm{a}}$ & $11^{\mathrm{a}}$ & $15^{\mathrm{a}}$ & $6^{\mathrm{a}}$ & $12^{\mathrm{a}}$ & $12^{\mathrm{a}}$ \\
\hline Pico do vírus & $34^{\mathrm{a}}$ & $23^{\mathrm{a}}$ & $25^{\mathrm{a}}$ & $23^{\mathrm{a}}$ & $14^{\mathrm{a}}$ & $20^{\mathrm{a}}$ & $21^{\mathrm{a}}$ \\
\hline Estabilização & $49^{\mathrm{a}}$ & $44^{\mathrm{a}}$ & $40^{\mathrm{a}}$ & $36^{\mathrm{a}}$ & $30^{\mathrm{a}}$ & $31^{\mathrm{a}}$ & $40^{\mathrm{a}}$ \\
\hline
\end{tabular}

Fonte: Dados compilados pelos autores através de registros do Ministério da Saúde (Brasil, 2009; Brasil, 2011; Brasil 2012a; Brasil, 2012b; Brasil, 2016; Brasil, 2018a; Brasil 2019a).

Em relação aos anos de 2014, 2015 e 2017, notou-se um número significativamente baixo de circulação do vírus Influenza A(H1N1)pdm09 em comparação com os demais anos já analisados. A surpresa maior foi o aumento de casos do vírus Influenza $\mathrm{A}(\mathrm{H} 3 \mathrm{~N} 2)$, seguindo um padrão de curva similar ao observado nos anos em que o predomínio foi do vírus Influenza A(H1N1)pdm09 (Gráfico 1) (Brasil, 2014; Brasil, 2015; Brasil, 2017). 
Gráfico 1. Comparativo entre os principais vírus identificados entre os anos de 2014 e 2019.

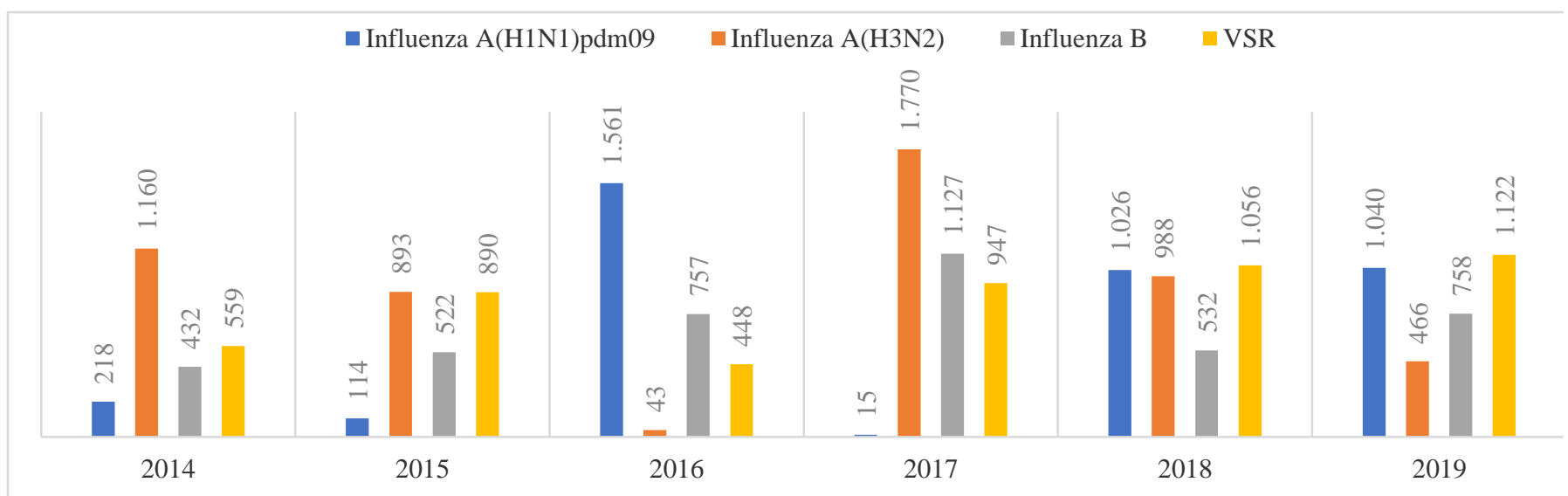

Legenda: VSR = outros vírus respiratórios.

Fonte: Dados compilados pelos autores através de registros do Ministério da Saúde (Brasil, 2014; Brasil, 2015; Brasil, 2016; Brasil, 2018a; Brasil 2019a).

É possível notar no Gráfico 1 que os anos de 2016 e 2017 tiveram resultados opostos em se tratando dos números relativos à Influenza A H1N1 e H3N2. Em 2016 houve um número bem elevado de casos de Influenza A(H1N1)pdm09, o que representou na época quase metade de todas as amostras positivas (45\% do total), e um número baixo de Influenza A(H3N2), menos de 1,3\% apenas. Em contrapartida, no ano de 2017, o número de amostras positivas para H1N1 foi de apenas 0,3\%, enquanto que o número de $\mathrm{H} 3 \mathrm{~N} 2$ representou $36 \%$.

Ademais, entre o grupo dos denominados "outros vírus respiratórios" (VSR, adenovírus e parainfluenza), o vírus sincicial predominou em todos os anos, inclusive sendo o de maior índice entre todos os vírus nos anos de 2018 e 2019. O período de maior ocorrência foi verificado na primeira metade do ano para o VSR e na segunda metade para o adenovírus e parainfluenza.

Quanto às regiões do país, o Norte e Centro Oeste, ao longo dos anos de 2016, 2017, 2018 e 2019, apresentaram um número de amostras positivas para Influenza A(H1N1)pdm09 muito baixo. Por outro lado, as regiões Sudeste e Sul tiveram os maiores índices, seguidos pelo Nordeste (Tabela 2). Cabe destacar que, o período de aumento gradativo do número de casos e pico de circulação do vírus nessas três regiões foram ligeiramente diferentes, com o Nordeste se antecipando quanto à circulação do vírus em relação aos outros.

A vigilância de influenza no Brasil compreende também o monitoramento da Síndrome Respiratória Aguda Grave (SRAG) em pacientes internados em Unidade de Terapia Intensiva (UTI) e pela vigilância universal de SRAG. Tendo em vista que esse último abrange todos os hospitalizados (quer estejam ou não em UTI) e óbitos, seus dados foram mais relevantes para o estudo e, por isso, a análise dos casos de pacientes internados exclusivamente em UTI não será realizada nesse trabalho.

É importante destacar que, os boletins epidemiológicos lançados entre os anos de 2009 e 2012 não seguiram um padrão de apresentação de dados que permitisse um comparativo integral entre eles próprios e entre os demais anos subsequentes (Brasil, 2009; Brasil, 2010; Brasil, 2011; Brasil 2012a; Brasil, 2012b). Dessa forma, inicialmente foi feita, nesta pesquisa, a exposição dos dados existentes desses anos. Em seguida, realizou-se um comparativo geral entre os anos de 2013 e 2019, incluindo todas as variáveis dos referidos boletins epidemiológicos, tendo em vista a coexistência delas em todos os anos a partir de 2013 (Brasil, 2013; Brasil, 2014; Brasil, 2015; Brasil, 2016; Brasil, 2017; Brasil, 2018a; Brasil 2019a). 
Tabela 2. Comparativo entre os períodos de aumento gradativo e pico do número de casos de Influenza A(H1N1)pdm09 das regiões Nordeste, Sudeste e Sul do Brasil.

\begin{tabular}{|c|c|c|c|c|c|c|}
\hline Anos & \multicolumn{2}{|c|}{2016} & \multicolumn{2}{|c|}{2018} & \multicolumn{2}{|c|}{2019} \\
\hline Regiões & $\begin{array}{l}\text { Início do } \\
\text { aumento } \\
\end{array}$ & Pico & $\begin{array}{l}\text { Início do } \\
\text { aumento } \\
\end{array}$ & Pico & $\begin{array}{l}\text { Início do } \\
\text { aumento } \\
\end{array}$ & Pico \\
\hline Nordeste & $5^{a}$ & $7^{\mathrm{a}}$ & $11^{\mathrm{a}}$ & $16^{\mathrm{a}}$ & $10^{\mathrm{a}}$ & $17^{\mathrm{a}}$ \\
\hline Sudeste & $8^{a}$ & $12^{a}$ & $16^{\mathrm{a}}$ & $24^{\mathrm{a}}$ & $13^{\mathrm{a}}$ & $20^{\mathrm{a}}$ \\
\hline Sul & $9^{a}$ & $15^{a}$ & $16^{\mathrm{a}}$ & $25^{\mathrm{a}}$ & $12^{\mathrm{a}}$ & $22^{a}$ \\
\hline
\end{tabular}

Fonte: Dados compilados pelos autores através de registros do Ministério da Saúde (Brasil, 2016; Brasil, 2018a; Brasil 2019a).

Para melhor análise estatística, foram escolhidos alguns estados que, em algum aspecto, se assemelham ao Maranhão: Ceará e Santa Catarina pelo número populacional; Goiás pela densidade demográfica; e Mato Grosso do Sul pela área. Piauí e Pará foram escolhidos pela proximidade geográfica e São Paulo e Paraná pelos maiores números, geralmente apresentados, de casos de influenza $\mathrm{A}(\mathrm{H} 1 \mathrm{~N} 1)$ pdm09 entre todos os anos estudados. Assim, cada região geográfica do país tem ao menos um estado como representante e, dessa forma, o estudo se torna mais abrangente.

Tabela 3. Casos de SRAG confirmados para influenza A(H1N1)pdm09 por região geográfica/UF de residência e ano de início dos sintomas. Brasil, 2009 - 2012.

\begin{tabular}{|c|c|c|c|c|c|c|c|c|}
\hline \multirow{3}{*}{ Estados/Regiões } & \multicolumn{8}{|c|}{ Anos } \\
\hline & \multicolumn{2}{|c|}{2009} & \multicolumn{2}{|c|}{2010} & \multicolumn{2}{|c|}{2011} & \multicolumn{2}{|c|}{2012} \\
\hline & $\mathrm{n}$ & $\%$ & $\mathrm{n}$ & $\%$ & $\mathrm{n}$ & $\%$ & $\mathrm{n}$ & $\%$ \\
\hline Maranhão & 48 & 48,5 & 66 & 55,5 & 0 & 0 & 0 & 0 \\
\hline Ceará & 47 & 28 & 11 & 14,7 & 0 & 0 & 53 & 13,8 \\
\hline Piauí & 51 & 24,5 & 37 & 27,2 & 0 & 0 & 1 & 3,1 \\
\hline Nordeste & 846 & 27,3 & 152 & 22,8 & 1 & 0,8 & 76 & 11,8 \\
\hline Pará & 477 & 36 & 281 & 34,8 & 0 & 0 & 3 & 1,8 \\
\hline Norte & 868 & 40,9 & 319 & 29,3 & 2 & 2,3 & 20 & 6,5 \\
\hline São Paulo & 7.407 & 37,7 & 108 & 3 & 28 & 2,9 & 370 & 8,4 \\
\hline Sudeste & 12.104 & 39 & 120 & 2,7 & 60 & 3 & 508 & 6,6 \\
\hline Goiás & 646 & 49,5 & 12 & 3,1 & 1 & 1,1 & 29 & 7,3 \\
\hline Mato Grosso do Sul & 89 & 27,6 & 0 & 0 & 3 & 6,8 & 60 & 21,9 \\
\hline Centro Oeste & 1.267 & 45,7 & 18 & 3,1 & 6 & 3,5 & 123 & 13 \\
\hline Santa Catarina & 2.155 & 44,7 & 20 & 3,5 & 7 & 2,7 & 743 & 23,1 \\
\hline Paraná & 30.650 & 76,6 & 344 & 21,9 & 2 & 0,2 & 621 & 15,9 \\
\hline Sul & 35.397 & 71,6 & 364 & 14,1 & 112 & 4,4 & 1.884 & 17 \\
\hline Brasil & 50.482 & 57,1 & 973 & 10,4 & 181 & 3,7 & 2.614 & 12,7 \\
\hline
\end{tabular}

Legenda: $\mathrm{n}=$ número de casos de SRAG por influenza A(H1N1)pdm09; \% = porcentagem de casos de SRAG por influenza A(H1N1)pdm09 em relação ao número total de casos de SRAG.

Fonte: Dados compilados pelos autores através de registros do Ministério da Saúde (Brasil, 2009; Brasil, 2010; Brasil, 2011; Brasil, 2012a; Brasil, 2012b).

Na Tabela 3, é possível verificar que o Maranhão foi o único estado que apresentou um aumento no número de casos entre os anos de 2009 e 2010, além de não ter identificado nenhum caso da doença no ano de 2011 e 2012 , acompanhando os estados de Roraima, Alagoas, Sergipe e Espírito Santo. De um modo geral, o Brasil teve 50.482 casos em 2009, apresentando uma redução significativa nos dois anos seguintes, (973 e 181 casos, respectivamente) e aumento para 2.614 casos de 2011 
para 2012. Os estados que obtiveram os maiores números de casos, foram os localizados nas regiões Sul e Sudeste (exceto Espírito Santo), sendo os principais Paraná e São Paulo. O Maranhão apresentou o $4^{\mathrm{a}}$ menor número entre os estados do Nordeste e o $10^{\mathrm{a}}$ menor do país, somado todo esse período.

Tabela 4. Distribuição dos óbitos de SRAG confirmados para influenza A/H1N1 2009 por região geográfica e ano de início dos sintomas.

\begin{tabular}{c|c|c|c|c|c|c}
\hline \multirow{2}{*}{ Anos } & \multicolumn{5}{|c|}{ Regiões } & \multirow{2}{*}{ Total de Letalidade } \\
\cline { 2 - 6 } & Norte & Nordeste & Centro Oeste & Sudeste & Sul & \\
\hline $\mathbf{2 0 0 9}$ & 50 & 62 & 167 & 992 & 789 & $\mathbf{2 . 0 6 0 ( 4 , 0 8 \% )}$ \\
\hline $\mathbf{2 0 1 0}$ & 48 & 23 & 4 & 17 & 21 & $\mathbf{1 1 3 ( 1 1 , 6 \% )}$ \\
\hline $\mathbf{2 0 1 1}$ & 0 & 0 & 1 & 6 & 14 & $\mathbf{2 1}(\mathbf{1 1 , 6 \% )}$ \\
\hline $\mathbf{2 0 1 2}$ & 7 & 12 & 25 & 118 & 189 & $\mathbf{3 5 1}(\mathbf{1 3 , 4 \% )}$ \\
\hline
\end{tabular}

Fonte: Dados compilados pelos autores através de registros do Ministério da Saúde (Brasil, 2009).

No que tange ao número de óbitos por influenza A(H1N1)pdm09 entre os anos de 2009 e 2012, não foram encontrados dados por estado, sendo esta uma limitação do estudo. Contudo, pelos dados contidos na Tabela 4, verifica-se que, assim como os maiores números de casos foram obtidos nas regiões Sul e Sudeste, os maiores números de óbitos também. Vale destacar que, entre os pacientes acometidos pela SRAG, em 2009 a taxa de letalidade foi de 4,08\%, em 2010 e 2011 de 11,6\%, e em 2012 de 13,4\%. As regiões Norte e Nordeste não tiveram nenhuma morte em 2011 (Brasil, 2009; Brasil, 2010; Brasil, 2011; Brasil, 2012).

A partir de 2013, os boletins epidemiológicos passaram a apresentar seus dados de forma padronizada, o que possibilitou uma melhor comparação entre os períodos. Dessa maneira, é possível apresentar os dados obtidos entre 2013 e 2019, sendo feitas as devidas comparações com os anos anteriores, sempre que necessário e possível (Brasil, 2013; Brasil, 2014; Brasil, 2015; Brasil, 2016; Brasil, 2017; Brasil, 2018a; Brasil 2019a).

Gráfico 2. Projeção das curvas de distribuição dos casos de SRAG hospitalizados confirmados para influenza A(H1N1)pdm09 por semana epidemiológica (SE). Brasil, 2013 - 2019.

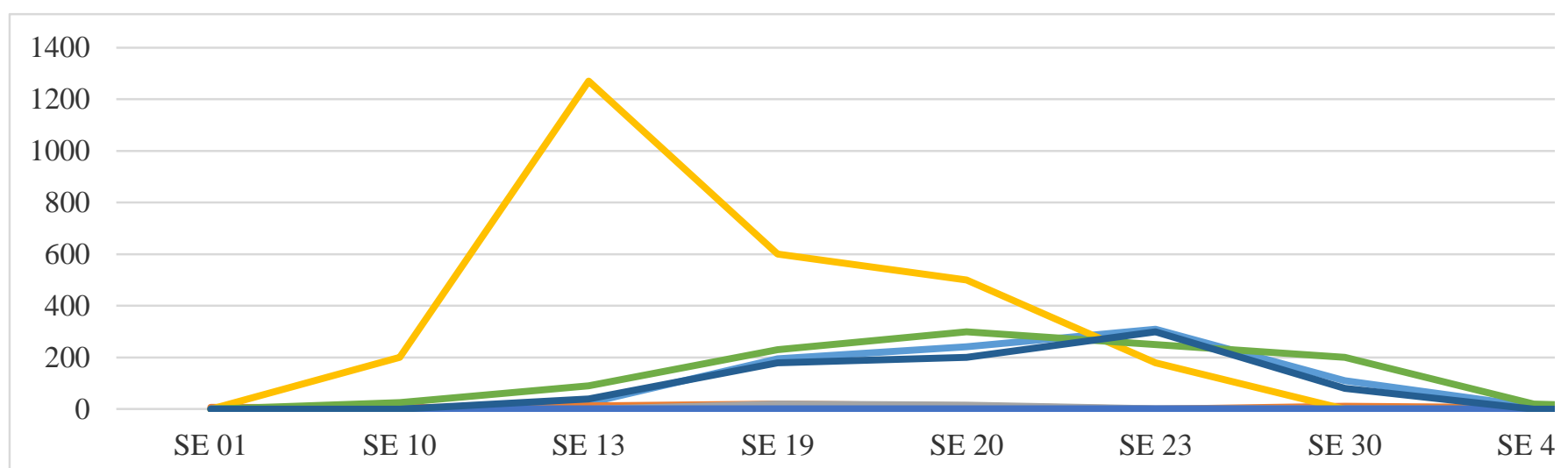

Legenda: SE = Semana Epidemiológica.

Fonte: Dados compilados pelos autores através de registros do Ministério da Saúde (Brasil, 2013; Brasil, 2014; Brasil, 2015; Brasil, 2016; Brasil, 2017; Brasil, 2018a; Brasil 2019a).

O Gráfico 2 aponta a distribuição dos casos de SRAG por influenza A(H1N1)pdm09 por semana epidemiológica, durante os anos de 2013 e 2019. Nele, pode-se observar três dinâmicas distintas da presença do vírus nos hospitalizados. Em 2014, 2015 e 2017, a média foi de 205 infectados apenas e a distribuição se deu o ano inteiro, sem que houvesse período com 
aumento gradativo do número de casos. Nos anos de 2013, 2018 e 2019, destaca-se a similaridade das curvas: início do aumento gradativo de casos por volta da $10^{\mathrm{a}} \mathrm{SE}$, pico entre a $20^{\mathrm{a}}-23^{\mathrm{a}} \mathrm{SE}$ e redução a partir da $40^{\mathrm{a}} \mathrm{SE}$. Já em 2016 , ocorreu uma antecipação da sazonalidade do vírus, com pico, inclusive, logo na $13^{\mathrm{a}} \mathrm{SE}$.

Através dos dados das Tabelas 3 e 5, constatou-se que, 76.509 pacientes desenvolveram a SRAG por influenza A(H1N1)pdm09, entre os anos de 2009 e 2019. Desses, 7.203 vieram a óbito, indicando uma taxa de letalidade de 9\%. Os anos de 2011, 2014, 2015 e 2017 representaram juntos apenas 1,04\% do total de casos e o ano de surgimento da pandemia, 65,98\%. Cabe destacar o ano de 2016 que, de maneira incomum, teve 13,88\% do total. Os demais anos tiveram a seguinte representação: 2010, 1,27\%; 2012, 3,41\%; 2013, 4,87\%; 2018, 5,07\% e 2019, 4,44\%.

$\mathrm{O}$ ano com maior número de mortes foi o de 2009, com um total de 2.060, seguido pelo ano de 2016, com 1.987. As maiores taxas de letalidade em pacientes com SRAG ocorreram justamente nos anos com menores números de casos: 2014 (34\%), 2015 (24\%) e 2017 (25\%). Além disso, os primeiros quatro anos (conforme Tabela 4) analisados foram os que apresentaram as menores taxas de letalidade: $2009,4,08 \% ; 2010,11,6 \% ; 2011,11,6 \%$; e 2012, 13,4\%.

O estado do Maranhão, ao longo de todo o período estudado, teve apenas $153(0,19 \%)$ casos de pacientes com SRAG por influenza A(H1N1)pdm09, a maioria em 2010 (66), seguido por 2009 (48) e 2018 (27). Em 2011, 2012, 2015 e 2019 não foram registrados nenhum caso. Em 2019, por exemplo, onde o número de casos e mortes foi bastante expressivo em todo o país, apenas o Maranhão não registrou infectados com o vírus influenza A(H1N1)pdm09.

Tendo como base o ano de 2016, onde ocorreu um surto inesperado de casos, em comparação com outros estados, o Maranhão registrou um número bem abaixo da média ( $\mathrm{n}=2 ; 0,01 \%$ dos casos de todo país). O estado de Goiás, que apresenta densidade demográfica equivalente ao do Maranhão, teve um número 183 vezes maior de casos $(n=366)$. Santa Catarina e Ceará, ambos com número de habitantes similares ao Maranhão, apresentaram, respectivamente, 710 e 93 casos. Piauí, que juntamente com o Maranhão possuem um dos menores Índices de Desenvolvimento Humano (IDH), registrou 15 casos. São Paulo e Paraná que apresentaram a maior incidência no ano, tiveram, respectivamente, 4.716 e 1.074 casos.

Dentre os óbitos por SRAG por influenza de 2013 a 2019 (Tabela 6), 70,3\% possuíam um fator ou condição de risco, tendo maior destaque os óbitos de adultos com mais de 60 anos (44,6\%), cardiopatas crônicos (29,7\%), diabetes mellitus $(21,5 \%)$ e pneumopatas $(20,7 \%)$. Além disso, 5.060 (73,4\%) fizeram uso de antiviral, com mediana de 4 dias entre os primeiros sintomas e o início do tratamento. Vale ressaltar que recomenda-se iniciar o tratamento nas primeiras 48 horas. Em alguns casos, pacientes receberam tratamento após 32, 64 e até 94 dias. Por fim, no que tange aos resultados encontrados, quanto à taxa de mortalidade para o Brasil, por 100.000 habitantes, houve uma variação entre os anos de: 0,16 em 2014; 0,08 em 2015; 1,07 em 2016; 0,24 em 2017; 0,66 em 2018 e 0,5 em 2019.

No presente estudo, após a apresentação e análise dos dados, três pontos demostraram ser muito relevantes e dignos de discussão: a sazonalidade do vírus influenza $\mathrm{A}(\mathrm{H} 1 \mathrm{~N} 1)$ pdm09; os fatores de risco que contribuem para o óbito; e a discrepância entre dados de estados com características semelhantes e possibilidade de subnotificação. 
Research, Society and Development, v. 10, n. 12, e453101219318, 2021

(CC BY 4.0) | ISSN 2525-3409 | DOI: http://dx.doi.org/10.33448/rsd-v10i12.19318

Tabela 5. Distribuição dos casos, óbitos e taxa de letalidade (t) por SRAG por influenza A(H1N1)pdm09 segundo região e unidade federativa. Brasil, 2013 - 2016.

\begin{tabular}{|c|c|c|c|c|c|c|c|c|c|c|c|c|c|c|}
\hline \multirow{3}{*}{$\begin{array}{l}\text { Estados/ } \\
\text { Regiões }\end{array}$} & \multicolumn{14}{|c|}{ Anos } \\
\hline & \multicolumn{2}{|c|}{2013} & \multicolumn{2}{|c|}{2014} & \multicolumn{2}{|c|}{2015} & \multicolumn{2}{|c|}{2016} & \multicolumn{2}{|c|}{2017} & \multicolumn{2}{|c|}{2018} & \multicolumn{2}{|c|}{ 2019* } \\
\hline & Casos & Óbitos (t) & Casos & Óbitos (t) & Casos & Óbitos (t) & Casos & Óbitos (t) & Casos & Óbitos (t) & Casos & Óbitos (t) & Casos & Óbitos (t) \\
\hline Maranhão & 1 & $0(0 \%)$ & 9 & $3(33 \%)$ & 0 & $0(0 \%)$ & 2 & $1(50 \%)$ & 0 & $0(0 \%)$ & 27 & $6(22 \%)$ & 0 & $0(0 \%)$ \\
\hline Ceará & 27 & $8(29 \%)$ & 11 & $0(0 \%)$ & 2 & $0(0 \%)$ & 93 & $14(15 \%)$ & 2 & $1(50 \%)$ & 257 & $58(22 \%)$ & 75 & $16(21 \%)$ \\
\hline Piauí & 9 & $4(44 \%)$ & 6 & $2(33 \%)$ & 0 & $0(0 \%)$ & 15 & $1(6 \%)$ & 0 & $0(0 \%)$ & 138 & $19(13 \%)$ & 2 & $0(0 \%)$ \\
\hline Nordeste & 116 & $29(25 \%)$ & 31 & $7(22 \%)$ & 2 & $0(0 \%)$ & 426 & $94(22 \%)$ & 10 & $4(40 \%)$ & 834 & $164(19 \%)$ & 302 & $72(23 \%)$ \\
\hline Pará & 97 & $19(19 \%)$ & 3 & $1(33 \%)$ & 2 & $0(0 \%)$ & 174 & $27(15 \%)$ & 0 & $0(0 \%)$ & 15 & $3(20 \%)$ & 29 & $5(17 \%)$ \\
\hline Norte & 114 & $27(23 \%)$ & 14 & $7(50 \%)$ & 2 & $0(0 \%)$ & 256 & $44(17 \%)$ & 0 & $0(0 \%)$ & 48 & $11(22 \%)$ & 171 & $44(25 \%)$ \\
\hline São Paulo & 1.972 & $405(20 \%)$ & 116 & $44(37 \%)$ & 18 & $7(38 \%)$ & 4.716 & $779(16 \%)$ & 30 & $6(20 \%)$ & 1.615 & $432(26 \%)$ & 557 & $127(22 \%)$ \\
\hline Sudeste & 2.466 & $537(21 \%)$ & 168 & $63(37 \%)$ & 24 & $9(37 \%)$ & 5.803 & $1.097(18 \%)$ & 34 & $8(23 \%)$ & 1.855 & $504(27 \%)$ & 901 & $239(26 \%)$ \\
\hline Goiás & 52 & $20(38 \%)$ & 32 & $14(43 \%)$ & 2 & $2(100 \%)$ & 366 & $88(24 \%)$ & 3 & $0(0 \%)$ & 345 & $68(19 \%)$ & 85 & $17(20 \%)$ \\
\hline $\begin{array}{c}\text { Mato Grosso } \\
\text { do Sul }\end{array}$ & 34 & $4(11 \%)$ & 53 & $21(39 \%)$ & 3 & $1(33 \%)$ & 474 & $95(20 \%)$ & 0 & $0(0 \%)$ & 50 & $12(24 \%)$ & 197 & $43(21 \%)$ \\
\hline Centro Oeste & 121 & $32(26 \%)$ & 150 & $58(38 \%)$ & 5 & $3(60 \%)$ & 1.041 & $218(20 \%)$ & 3 & $0(0 \%)$ & 493 & $93(18 \%)$ & 387 & $81(20 \%)$ \\
\hline $\begin{array}{c}\text { Santa } \\
\text { Catarina }\end{array}$ & 225 & $34(15 \%)$ & 19 & $4(21 \%)$ & 55 & $13(23 \%)$ & 710 & $112(15 \%)$ & 0 & $0(0 \%)$ & 159 & $35(22 \%)$ & 226 & $37(16 \%)$ \\
\hline Paraná & 353 & $47(13 \%)$ & 43 & $8(18 \%)$ & 31 & $4(12 \%)$ & 1.074 & $216(20 \%)$ & 0 & $0(0 \%)$ & 237 & $46(19 \%)$ & 431 & $80(18 \%)$ \\
\hline Sul & 911 & $139(15 \%)$ & 91 & $23(25 \%)$ & 86 & $17(19 \%)$ & 3.092 & $533(17 \%)$ & 1 & 0 & 647 & $143(22 \%)$ & 771 & $136(17 \%)$ \\
\hline Brasil & 3.733 & $768(20 \%)$ & 455 & $158(34 \%)$ & 119 & $29(24 \%)$ & 10.625 & $1.987(18 \%)$ & 48 & $12(25 \%)$ & 3.880 & $917(23 \%)$ & 3.399 & $787(23 \%)$ \\
\hline
\end{tabular}

Legenda: *Somente o número total de casos no Brasil está atualizado até a última SE do ano. Os demais dados por estado estão atualizados somente até a $32^{\text {a }}$ SE.

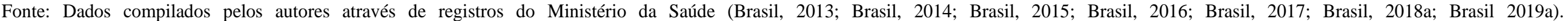


Tabela 6. Distribuição dos óbitos de SRAG por influenza, segundo fator de risco e utilização de antiviral. Brasil, 2013 - 2019.

\begin{tabular}{|c|c|c|c|c|c|c|c|c|c|c|c|c|c|c|}
\hline \multirow{3}{*}{ Distribuição } & \multicolumn{14}{|c|}{ Anos } \\
\hline & \multicolumn{2}{|c|}{2013} & \multicolumn{2}{|c|}{2014} & \multicolumn{2}{|c|}{2015} & \multicolumn{2}{|c|}{2016} & \multicolumn{2}{|c|}{2017} & \multicolumn{2}{|c|}{2018} & \multicolumn{2}{|c|}{2019} \\
\hline & $\mathrm{n}$ & $\%$ & $\mathrm{n}$ & $\%$ & $\mathrm{n}$ & $\%$ & $\mathrm{n}$ & $\%$ & $\mathrm{n}$ & $\%$ & $\mathrm{n}$ & $\%$ & $\mathrm{n}$ & $\%$ \\
\hline $\begin{array}{c}\text { Adultos } \\
>60 \text { anos }\end{array}$ & 223 & 23,4 & 92 & 28,9 & 68 & 42,5 & 653 & 42,2 & 258 & 65,6 & 581 & 55,1 & 441 & 54,6 \\
\hline $\begin{array}{c}\text { Doença } \\
\text { cardiovas-cular } \\
\text { crônica }\end{array}$ & 198 & 20,7 & 62 & 19,5 & 46 & 28,8 & 455 & 29,4 & 166 & 42,2 & 333 & 31,6 & 290 & 35,9 \\
\hline $\begin{array}{l}\text { Diabetes } \\
\text { Mellitus }\end{array}$ & 154 & 16,1 & 41 & 12,9 & 29 & 18,1 & 365 & 23,6 & 115 & 29,3 & 242 & 22,9 & 223 & 27,6 \\
\hline $\begin{array}{c}\text { Pneumopa-tias } \\
\text { crônicas }\end{array}$ & 139 & 14,6 & 51 & 16 & 31 & 19,4 & 360 & 23,2 & 119 & 30,3 & 260 & 24,6 & 137 & 17 \\
\hline Obesidade & 119 & 12,5 & 39 & 12,3 & 12 & 7,5 & 262 & 16,9 & 41 & 10,4 & 154 & 14,6 & 80 & 9,9 \\
\hline $\begin{array}{c}\text { Imuno- } \\
\text { deficiência }\end{array}$ & 90 & 9,4 & 18 & 5,7 & 6 & 3,8 & 142 & 9,2 & 32 & 8,1 & 88 & 8,3 & 74 & 9,2 \\
\hline $\begin{array}{c}\text { Doença renal } \\
\text { crônica }\end{array}$ & 54 & 5,7 & 14 & 4,4 & 8 & 5 & 111 & 7,2 & 30 & 7,6 & 99 & 9,4 & 70 & 8,7 \\
\hline $\begin{array}{c}\text { Doença } \\
\text { neurológica } \\
\text { crônica } \\
\end{array}$ & 44 & 4,6 & 16 & 5 & 10 & 6,3 & 116 & 7,5 & 38 & 9,7 & 111 & 10,5 & 69 & 8,5 \\
\hline $\begin{array}{c}\text { Crianças } \\
<2 \text { anos } \\
\end{array}$ & 36 & 3,8 & 12 & 3,8 & 2 & 1,3 & - & - & - & - & - & - & - & - \\
\hline $\begin{array}{c}\text { Crianças } \\
<5 \text { anos }\end{array}$ & - & - & - & - & - & - & 159 & 10,3 & 28 & 7,1 & 98 & 9,3 & 95 & 11,8 \\
\hline $\begin{array}{c}\text { Doença hepática } \\
\text { crônica }\end{array}$ & 22 & 2,3 & 7 & 2,2 & 3 & 1,9 & 48 & 3,1 & 13 & 3,3 & 26 & 2,5 & 21 & 2,6 \\
\hline $\begin{array}{c}\text { Síndrome de } \\
\text { Down }\end{array}$ & 18 & 1,9 & 1 & 0,3 & - & - & 19 & 1,2 & 8 & 2 & 13 & 1,2 & 11 & 1,4 \\
\hline Gestantes & 16 & 1,7 & 6 & 1,9 & 5 & 3,1 & 29 & 1,9 & 4 & 1 & 16 & 1,5 & 12 & 1,5 \\
\hline $\begin{array}{c}\text { Puerpério } \\
\text { (até } 42 \text { dias) }\end{array}$ & 3 & 0,3 & 1 & 0,3 & 1 & 0,6 & 8 & 0,5 & 2 & 0,5 & 3 & 0,3 & 2 & 0,2 \\
\hline Indígenas & 2 & 0,2 & 5 & 1,6 & 1 & 0,6 & 19 & 1,2 & - & - & 3 & 0,3 & 5 & 0,6 \\
\hline $\begin{array}{c}\text { Com fatores de } \\
\text { risco }\end{array}$ & \multicolumn{2}{|c|}{$\begin{array}{c}621 \\
(65 \%) \\
\end{array}$} & \multicolumn{2}{|c|}{$204(64,2)$} & \multicolumn{2}{|c|}{$\begin{array}{c}104 \\
(65 \%) \\
\end{array}$} & \multicolumn{2}{|c|}{$\begin{array}{c}1.549 \\
(69,8 \%) \\
\end{array}$} & \multicolumn{2}{|c|}{$\begin{array}{c}1 \\
393 \\
(78,9 \%) \\
\end{array}$} & \multicolumn{2}{|c|}{$\begin{array}{c}1.055 \\
(76,4 \%) \\
\end{array}$} & \multicolumn{2}{|c|}{$\begin{array}{c}808 \\
(72,9 \%) \\
\end{array}$} \\
\hline $\begin{array}{c}\text { Que utilizaram } \\
\text { antiviral }\end{array}$ & \multicolumn{2}{|c|}{$\begin{array}{c}759 \\
(79,5 \%)\end{array}$} & \multicolumn{2}{|c|}{$\begin{array}{c}223 \\
(70,1 \%)\end{array}$} & \multicolumn{2}{|c|}{$\begin{array}{c}103 \\
(64,4 \%)\end{array}$} & \multicolumn{2}{|c|}{$\begin{array}{c}1.711 \\
(77,1 \%)\end{array}$} & \multicolumn{2}{|c|}{$\begin{array}{c}343 \\
(68,9 \%)\end{array}$} & \multicolumn{2}{|c|}{$\begin{array}{l}1.077 \\
(78 \%)\end{array}$} & \multicolumn{2}{|c|}{$\begin{array}{c}844 \\
(76,1 \%)\end{array}$} \\
\hline
\end{tabular}

Fonte: Dados compilados pelos autores através de registros do Ministério da Saúde (Brasil, 2013; Brasil, 2014; Brasil, 2015; Brasil, 2016; Brasil, 2017; Brasil, 2018a; Brasil 2019a).

Inicialmente, é possível observar e confirmar o comportamento sazonal do vírus influenza, mas com algumas ressalvas que são importantes para as decisões das autoridades públicas no controle das infecções ano a ano.

A questão da sazonalidade da influenza e de outros vírus respiratórios ainda não é bem compreendida e são poucos os estudos à respeito, restando apenas o conhecimento de sua existência. No entanto, Alonso et al. (2007) já sugeria que “o clima pode ter uma influência direta na sobrevida do vírus, na eficiência da transmissão, na suscetibilidade do hospedeiro, além de proporcionar a aglomeração da população".

Para Almeida et al., (2015), em áreas de clima temperado, a sazonalidade é bem definida e seu pico de incidência ocorre durante o inverno. Além disso, para o autor, no Hemisfério Norte a gripe ocorre entre os meses de outubro e abril de cada ano; no Hemisfério Sul, entre abril e setembro.

Em estudo mais recente e amplo, Almeida (2018) concorda que em climas temperados a sazonalidade é bem definida. Entretanto, acrescenta que nos países de clima tropical, onde a temperatura tem pouca oscilação e a umidade prevalece o ano 
todo, é difícil se estabelecer um padrão. No Brasil, por exemplo, em virtude de sua dimensão continental, apesar de ser considerado um país tropical, engloba uma ampla gama de latitudes e seis subtipos climáticos, e assim sendo, acaba por experimentar comportamentos diferentes do mesmo vírus nos extremos do seu território: ao Sul e Sudeste, de clima temperado, o comportamento é um; ao Norte e Nordeste, de clima tropical, outro (Almeida, 2018).

No estudo de Almeida (2018), apenas 44\% dos estados apresentaram uma periocidade anual, principalmente aqueles localizados na costa e mais ao sul do país. Nesses estados, denominados de sazonais, a gripe começa na região Nordeste, espalhando-se para o Sul. Segundo a mesma pesquisa, a onda se inicia no Ceará, um pouco antes, com pico por volta da $15^{\mathrm{a}}$ semana, e segue para a região Sul, na qual o pico ocorre por volta da $21^{\text {a }}$ SE. Vale frisar que não apenas o clima e a localização, mas a infraestrutura rodoviária desempenha um papel importante na sazonalidade da influenza e na sua propagação. As regiões Norte e Centro Oeste, devido ao isolamento geográfico, acabam por se desconectarem dessa onda surgida no Nordeste (Almeida, 2018).

No presente trabalho, conforme a Tabela 2, é possível observar que na região Nordeste, o aumento gradativo dos casos e o pico de circulação sempre se iniciam antes das regiões Sul e Sudeste. Além disso, em todo o período estudado, as regiões Norte e Centro Oeste apresentaram poucos casos. Ambas constatações vão ao encontro do estudo de Almeida (2018), que afirma: "uma campanha de vacinação de calendário único, com o objetivo de cobrir todo o país, requer esforços quase continentais". Assim sendo, entende-se que o calendário comum de vacinação poderia ser otimizado a partir da identificação de estados onde um cronograma mais cedo fosse desejável e de outros em que um adiamento não fosse prejudicial.

No que tange aos fatores de risco mais comumentes encontrados em pacientes que evoluíram a óbito por influenza, quatro deles se destacaram: adultos > 60 anos, cardiopatas, pneumopatas e diabetes mellitus (Tabela 6).

Estudos realizados anteriormente, logo após o período pandêmico, já indicavam a idade como fator de risco importante. Louie et al., (2011) mostraram que na Califórnia a taxa de mortalidade foi maior em pacientes com idade superior a 50 anos. Wilking et al., (2010) também identificaram que na Alemanha, pacientes com idade igual ou superior a 60 anos apresentaram as maiores taxas de mortalidade. No Brasil não foi diferente e o maior número de mortes foi entre os idosos a partir de 60 anos (Tabela 6). Louie, et al., (2011) afirmaram ainda que "adultos com mais de 60 anos podem ter sido poupados devido à imunidade relativa pré-existente. No entanto, uma vez infectado (...), as taxas de letalidade eram altas especialmente em idosos".

Dessa forma, os idosos necessitam de maiores cuidados com a prevenção, sendo essa tarefa de responsabilidade das pessoas de sua convivência e dos profissionais de saúde que acabam tendo que oferecer seus serviços em unidades de saúde, em virtude de condições diversas que acometem esse grupo de risco. Preocupado com esta população, o estado de São Paulo já havia sancionado a lei $\mathrm{n}^{\circ}$ 10.003, de 24 de julho de 1998, que instituia a lei da vacinação do idoso, a fim de garantir a este público, além da vacina contra o vírus influenza, também a vacina dupla adulto (contra tétano e difteria) e a que previne o pneumococo, bactéria causadora de pneumonias, otites, sinusites, faringites e meningites; tendo sido revogada pela lei $\mathrm{n}^{\circ}$ 12.548, de 27de fevereiro de 2007, cujo direito se manteve no artigo 14, parágrafo $1^{\circ}$ (Estado de São Paulo, 1998; Estado de São Paulo, 2007). Mas o Estatuto do Idoso (Brasil, 2003), neste ínterem, em seu artigo $3^{\circ}$, consolidou como "obrigação da família, da comunidade, da sociedade e do Poder Público assegurar ao idoso, com absoluta prioridade, a efetivação do direito à vida, à saúde..." O que justifica a responsabilidade das pessoas da conviênvia dos idosos e dos profissionais de saúde no que tange a maiores cuidados de prevenção aos idosos.

Por outro lado, Lenzi et al., (2013) demostraram que, em 2009, o maior percentual de óbitos ocorreu no grupo de pacientes hospitalizados e a razão deveu-se porque estes pacientes hospitalizados já se encontravam clinicamente graves: "a taxa de óbito foi significativamente maior nos pacientes que apresentavam outras comorbidades associadas à infecção pela Influenza Pandêmica A(H1N1)2009 (...) mesmo na vigência do uso do antiviral Oseltamivir” (Lenzi et al., 2013). 
Goldstein, et al., (2010) constataram que o tempo de início do tratamento com Oseltamivir é relevante para cura do paciente. Naqueles que iniciaram o tratamento em até dois dias após início dos sintomas, houve uma redução em $42 \%$ da probabilidade de ocorrência de uma ou mais infecções secundárias. O Ministério da Saúde (Brasil, 2017), em seu protocolo de tratamento, indica que estudos observacionais demonstraram maior benefício do fosfato de oseltamivir quando iniciado em até 48 horas do início dos sintomas. Contudo, também sugere que há benefícios se iniciado de quatro a cinco dias do início do quadro clínico.

Dentro desse contexto de pacientes que desenvolvem a doença e tenham fatores de risco associados, o Ministério da Saúde (Brasil, 2020a), durante as campanhas de vacinação, já os contempla como grupos prioritários para receberem a vacina, estando eles incluídos logo nas primeiras duas fases: idosos no primeiro mês e pacientes com doenças crônicas já no segundo. Contudo, ainda hoje "mantém-se a necessidade de prescrição médica especificando o motivo da indicação da vacina, que deverá ser apresentada no ato da vacinação" (Brasil, 2020a).

Indubitavelmente, tal comprovação precisa ser realizada, afim de que apenas aqueles que necessitem se antecipar na imunização sejam contemplados. Entretanto, é natural que existam pessoas já diagnosticadas com tais doença que não fazem o acompanhamento adequado e, exigir a prescrição médica das mesmas, pode se tornar um entrave maior para a vacinação. Apesar de que os pacientes cadastrados em programas de controle das doenças crônicas do Sistema Único de Saúde (SUS), possam se dirigir aos postos onde são acompanhados para receberem a vacina, nos casos em que a unidade não possua setor específico de vacinação, aquela exigência se tornaria necessária e o embaraço permaneceria.

Por fim, identificou-se com os resultados, uma certa discrepância de informações. O estado do Maranhão, localizado na mesma região e próximo geograficamente aos estados do Ceará e Piauí, apresentou números bem abaixo desses e, também, consideravelmente abaixo de outros estados do país, cujas características similares ao do Maranhão (número de habitantes, densidade demográfica, área) poderiam indicar dados mais parecidos (Tabela 5).

Diversas explicações poderiam justificar tal realidade. Primeiro, pode ser mencionado o fato de que, naturalmente, a região Nordeste já apresenta menos casos, por apresentar um clima tropical que não favorece os aspectos da sazonalidade, como já discutido. Além disso, Almeida (2018) constatou, em seu estudo, o início da onda no Ceará, não indicando o trajeto pelo Maranhão. Entretanto, se fossem tomadas como base tais afirmações, o estado do Piauí também não apresentaria um número de casos tão superior assim, tendo em vista que estaria numa posição igual ao do Maranhão.

Uma segunda explicação poderia estar relacionada à cobertura vacinal dos estados. Todavia, segundo dados do Sistema de Informações do Programa Nacional de Imunizações (SIPNI), no ano de 2019, por exemplo, apenas seis estados da federação alcançaram menos do que $90 \%$ das metas exigidas, nenhum deles com cobertura vacinal menor que 86\% (Brasil, 2020b).

Outro motivo poderia ser o fato de que o Estado do Maranhão, apesar de possuir um aeroporto internacional, não tem muito fluxo de turistas e circulação de pessoas no seu território, dificultando assim a propagação do vírus (IBGE, 2010). Contudo, o estado do Goiás, que possui densidade demográfica muito próxima ao do estado do Maranhão (IBGE, 2010) e encontra-se na região Centro Oeste, não poderia apresentar dados tão discrepantes, pois tal região, juntamente com o Norte, encontra-se geograficamente isolada das demais (Almeida, 2018).

Em último caso, poderia se pensar em subnotificação e, observando os Boletins Epidemiológicos do Ministério da Saúde sobre a Influenza de 2013 e 2019, é possível verificar uma redução significativa de unidades sentinelas (US), as quais seriam responsáveis por identificar os vírus respiratórios circulantes e monitorar a demanda de atendimento dos casos hospitalizados e óbitos. Em 2013 eram 219 US e em 2019, apenas 163 - demostrando uma redução de 25,5\% (Brasil, 2013; Brasil, 2019). 
Em 2019, das 163 unidades sentinelas cadastradas, 30\% sequer reportaram os dados ao Ministério. Além disso, das $114(70 \%)$ que reportaram os dados, " $58,7 \%$ (70/114) US possuem indicador de coleta entre 80 a 100\% que é recomendado; 21,1\% (24/114) US possuem indicador entre 50 a 79\%; e, 20,2\% (23/114) US possuem indicador de coleta de amostras entre 0,5 a 49\%" (Brasil, 2019). Neste sentido, chama atenção a afirmação do MS registrada no Boletim, de que "as regiões Norte e Nordeste necessitam de ações de fortalecimento nas atividades de vigilância sentinela da SG e assim apresentarem dados mais representativos para a vigilância da influenza no Brasil" (Brasil, 2019a).

Outro aspecto que poderia sugerir subnotificação é que, de toda região Nordeste, apenas os estados do Maranhão e Alagoas possuem, cada, somente uma unidade sentinela registrada. O hospital da criança Dr. Odorico Amaral de Matos, localizado na capital maranhense, possui o terceiro pior indicador de repasse de dados (30,2\%) (Brasil, 2019a). Ademais, Carvalho, Dourado \& Bierrenbach (2011) afirmam que a subnotificação de doenças de notificação compulsória acontece quando um caso simplesmente não é notificado ou ocorre fora do período estabelecido. Acrescenta ainda que estimativas equivocadas podem decorrer disso, o que prejudica o planejamento de estratégias de prevenção e controle, podendo levar à sublocação de ações e recursos.

Ferreira, Portela e Vasconcellos (2000) corroboram dizendo que "o sistema de vigilância epidemiológica de doenças transmissíveis no Brasil é rotineiramente passivo". Fatores que podem contribuir para a subnotificação de casos podem ser: o desconhecimento das doenças de notificação compulsória, como notificar, mudanças na definição de caso e a crença de que outro profissional notificou o caso (Ferreira et al., 2000). Tal fato contribui para que não haja a efetiva atuação do estado em implementar e executar políticas econômicas e sociais "que visem à redução de riscos de doenças e de outros agravos e no estabelecimento de condições que assegurem acesso universal e igualitário às ações e aos serviços para a sua promoção, proteção e recuperação" (Brasil, 1990). Ações que visem fortalecer o sistema de notificação devem ser implementadas, bem como a ampliação de políticas públicas voltadas para a saúde, incluindo educação em saúde, a fim de reduzir agravos, em especial de doenças passíveis de prevenção e controle.

\section{Conclusão}

Com a pandemia do Covid-19, as autoridades públicas abriram o alerta para o aumento inesperado de casos de gripe A(H1N1)pdm09, já no início do ano 2020. O pânico provocado na população pode ter induzido a um maior número de pessoas em busca de atendimento médico e, tendo em vista que as doenças apresentam um quadro clínico muito semelhantes, estratégias como a antecipação do calendário vacinal de influenza e testes em massa foram realizadas para enfrentar o novo vírus.

Na década em estudo, foram notificados 76.509 casos de SRAG por influenza A(H1N1)pdm09 no Brasil, 2.796 no Nordeste e 153 casos no Maranhão. Desses, no país inteiro, 7.203 foram a óbito, sendo 467 do Nordeste. Os maiores registros de casos e mortes foram observados nas regiões Sul e Sudeste do país, mais especificamente nos estados do Paraná e São Paulo.

A circulação do vírus influenza A(H1N1)pdm09 ocorre de forma sazonal e bem delimitada em locais de clima temperado, enquanto que tal aspecto não é observado em áreas de clima tropical. Devido à grande extensão do Brasil, nos seus extremos o vírus acaba se comportando de maneira diferente e essa característica se torna relevante para compreender a dinâmica. Inicialmente, a circulação ocorre no Nordeste e segue para a região Sul. O Norte e Centro Oeste encontram-se desconectados desse trajeto e acabam registrando o menor número de casos.

Dentre os fatores de risco mais comumentes encontrados em pacientes que evoluíram a óbito por influenza $\mathrm{A}(\mathrm{H} 1 \mathrm{N1}$ )pdm09, quatro deles se destacaram: adultos > 60 anos, cardiopatas, pneumopatas e diabetes mellitus. Reconhecer esses grupos, permite um cuidado maior na prevenção e tratamento precoce, reduzindo o número de casos e aumentando a taxa 
de cura, respectivamente.

Quanto à vacinação, verificou-se a necessidade de melhor adequação das campanhas, respeitando o período de pico de epidemia em cada região, flexibilizando assim as datas. Além disso, no que tange à cobertura vacinal dos grupos prioritários de pacientes com doenças crônicas, evidenciou-se a necessidade de identificação dessas pessoas em algum sistema ou através de documento oficial, constando a informação sobre sua patologia, afim de que possam receber a vacina e até mesmo orientações de cuidado gerais em qualquer posto de vacinação, sem que fosse exigida a prescrição médica.

Os dados encontrados nos Boletins Epidemiológicos sugeriram a subnotificação dos casos na região Norte e Nordeste do país, mais especificamente no estado do Maranhão. Dessa forma, o aumento no número de unidades sentinelas e melhor distribuição, favoreceria o monitoramento e, consequentemente, proporcionaria a orientação adequada ao Ministério da Saúde e Secretarias de Saúde Estaduais e Municipais na tomada de decisões frente às epidemias, consolidando o que afirmam Constituição Federal, Lei 8080/90 e outras que dizem respeito à saúde da população. A partir do momento em que se tem conhecimento de um novo vírus circulando no território nacional ou a mudança no padrão de sazonalidade de vírus já conhecidos, as autoridades públicas podem antecipar suas ações e adequar as estratégias para o enfrentamento das adversidades, sem que permita à população entrar em pânico.

Em virtude da importância da temática avaliada, sugere-se que periodicamente novas pesquisas venham a público com o intuito de atualização constante dos levantamentos pontuados, favorecendo difusão técnico-científica e, ainda, ações de políticas públicas mais efetivas.

\section{Referências}

Almeida, A. R. M. D. (2018). Dinâmica sazonal da influenza no Brasil: a importância da latitude e do clima (Tese de Doutorado, Oswaldo Cruz).

Almeida, F. J., Berezin, E. N., Farhat, C. K., Cintra, O. A., Stein, R. T., Burns, D. A., Arns, C. C., Lomar, A. V., Toniolo-Neto, J., \& Medeiros, R. (2015). Consenso para o tratamento e profilaxia da influenza (Gripe) no Brasil. Sociedade brasileira de pediatria.

Alonso, W. J., Viboud, C., Simonsen, L., Hirano, E. W., Daufenbach, L. Z., \& Miller, M. A. (2007). Seasonality of influenza in Brazil: a traveling wave from the Amazon to the subtropics. American journal of epidemiology, 165(12), 1434-1442.

Brasil (1988). Presidência da República. Casa Civil. Constituição da República Federativa do Brasil de 1988. http://www.planalto.gov.br/ccivil_03/constituicao/constituicao.htm.

Brasil (1990). Lei 8080 de 19 de setembro de 1990. Dispõe sobre as condições para a promoção, proteção e recuperação da saúde, a organização e o funcionamento dos serviços correspondentes e dá outras providências. http://www.planalto.gov.br/ccivil_03/leis/l8080.htm

Brasil (2003). Presidência da República. Casa Civil. Lei nº 10.741, de $1^{\text {o }}$ de outubro de 2003. Dispõe sobre o Estatuto do Idoso e dá outras providências.

Brasil (2009). Ministério da Saúde. Secretaria de Vigilância em Saúde. Informe técnico de influenza. Brasília: Ministério da Saúde.

Brasil (2011). Ministério da Saúde. Secretaria de Vigilância em Saúde. Informe técnico de influenza. Brasília: Ministério da Saúde.

Brasil (2012a). Ministério da Saúde. Secretaria de Vigilância em Saúde. Informe técnico de influenza. Brasília: Ministério da Saúde.

Brasil (2012b). Ministério da Saúde. Secretaria de Vigilância em Saúde. Informe técnico de influenza. Brasília: Ministério da Saúde.

Brasil (2013). Ministério da Saúde. Secretaria de Vigilância em Saúde. Informe Epidemiológico - Influenza SE 52. Brasília: Ministério da Saúde.

Brasil (2014). Ministério da Saúde. Secretaria de Vigilância em Saúde. Boletim Epidemiológico - Influenza SE 50. Brasília: Ministério da Saúde.

Brasil (2015). Ministério da Saúde. Secretaria de Vigilância em Saúde. Informe Epidemiológico - Influenza SE 47. Brasília: Ministério da Saúde.

Brasil (2016). Ministério da Saúde. Secretaria de Vigilância em Saúde. Informe Epidemiológico - Influenza SE 52. Brasília: Ministério da Saúde.

Brasil (2017). Ministério da Saúde. Secretaria de Vigilância em Saúde. Informe Epidemiológico - Influenza SE 52. Brasília: Ministério da Saúde.

Brasil (2018a). Ministério da Saúde. Secretaria de Vigilância em Saúde. Informe Epidemiológico - Influenza SE 52. Brasília: Ministério da Saúde.

Brasil (2018b). Protocolo de tratamento de Influenza: 2017. Ministério da Saúde, Secretaria de Vigilância em Saúde, Departamento de Vigilância das Doenças Transmissíveis. Brasília: Ministério da Saúde.

Brasil (2019a). Ministério da Saúde. Secretaria de Vigilância em Saúde. Boletim Epidemiológico SE 49. Brasília: Ministério da Saúde. 
Brasil (2019b). Guia de vigilância em saúde: volume único. Ministério da Saúde, Secretaria de Vigilância em Saúde, Coordenação-Geral de Desenvolvimento da Epidemiologia em Serviços. Brasília: Ministério da Saúde.

Brasil (2020a). Informe técnico vacinação: 22 $2^{\mathrm{a}}$ campanha nacional de vacinação contra a influenza. Brasília: Ministério da Saúde, 2020. https://www.saude.gov.br/images/pdf/2020/marco/30/GRIPE-Informe-Tecnico-Influenza--final-2.pdf>.

Brasil (2020a). Informe técnico vacinação: 22a campanha nacional de vacinação contra a influenza. Brasília: Ministério da Saúde, 2020.

Brasil (2020b). Ministério da Saúde. Sistema de Informações do Programa Nacional de Imunizações (SIPNI). Vacinômetro Influenza. DATASUS. Brasília: Ministério da Saúde, 2020.

Carvalho, C. N., Dourado, I., \& Bierrenbach, A. L. (2011). Subnotificação da comorbidade tuberculose e aids: uma aplicação do método de linkage. Revista de Saúde Pública, 45, 548-555.

Coimbra, L. C. (2010). Saiba mais sobre a gripe A. Universidade Federal do Maranhão, Maranhão. https://portais.ufma.br/PortalUfma/paginas/noticias/noticia.jsf?id=7846>.

Estado de São Paulo (1998). Lei $\mathrm{n}^{\circ}$ 10.003, de 24 de junho de 1998. Institui o Programa Estadual de Vacinação da Terceira Idade. https://www.al.sp.gov.br/repositorio/legislacao/lei/1998/lei-10003-24.06.1998.html.

Estado de São Paulo (2007). Lei $\mathrm{n}^{\mathrm{o}}$ 12.548, de 27 de fevereiro de 2007. Consolida a legislação relativa ao idoso. https://www.al.sp.gov.br/repositorio/legislacao/lei/2007/lei-12548-27.02.2007.html.

Estado do Maranhão (2020). Decreto n ${ }^{\circ} 35.672$, de 19 de março de 2020. Declara situação de calamidade no Estado do Maranhão em virtude do aumento do número de infecções pelo vírus H1N1, da existência de casos suspeitos de contaminação pela COVID-19.

Estrela, C. (2018). Metodologia científica: ciência, ensino, pesquisa. Artes Médicas.

Felinto, G. M., Escosteguy, C. C., \& Medronho, R. D. A. (2018). Fatores associados ao óbito dos casos graves de influenza A (H1N1) pdm09. Cadernos Saúde Coletiva, 27, 11-19.

Ferreira, V., Portela, M. C., \& Vasconcellos, M. T. (2000). Fatores associados à subnotificação de pacientes com Aids, no Rio de Janeiro, RJ, 1996. Revista de Saúde Pública, 34, 170-177.

Goldstein, E., Cowling, B. J., O'Hagan, J. J., Danon, L., Fang, V. J., Hagy, A., Miller, J. C., Reshef, D. Robins, J., Biedrzycki, P., \& Lipsitch, M. (2010). Oseltamivir for treatment and prevention of pandemic influenza A/H1N1 virus infection in households, Milwaukee, 2009. BMC infectious diseases, 10(1), 1-7.

IBGE (2010). Censo Demográfico IBGE 2010: Resultados Gerais da Amostra. <https://censo2010.ibge.gov.br/resultados.html>

Lenzi, L., Mello, Â. M. D., Silva, L. R. D., Grochocki, M. H. C., \& Pontarolo, R. (2012). Pandemic influenza A (H1N1) 2009: risk factors for hospitalization. Jornal Brasileiro de Pneumologia, 38, 57-65.

Lima-Costa, M. F., \& Barreto, S. M. (2003). Tipos de estudos epidemiológicos: conceitos básicos e aplicações na área do envelhecimento. Epidemiologia e serviços de saúde, 12(4), 189-201.

Lobo, R. D. (2015). Fatores de risco para aquisição de influenza A (H1N1) pdm09 entre os profissionais de saúde (Tese de Doutorado, Universidade de São Paulo).

Louie, J. K., Jean, C., Acosta, M., Samuel, M. C., Matyas, B. T., \& Schechter, R. (2011). A review of adult mortality due to 2009 pandemic (H1N1) influenza A in California. PLoS one, 6(4), e18221.

Opendatasus (2021). SRAG 2021 - Banco de Dados de Síndrome Respiratória Aguda Grave - incluindo dados da COVID-19. Vigilância de Síndrome Respiratória Aguda Grave (SRAG). https://opendatasus.saude.gov.br/dataset/bd-srag-2021\#: :text=Atualmente\%2C\%20o\%20sistema\%20oficial\%20 para,Gripe\%20(SI VEP\%2DGripe.

Silva Júnior, S. H. A. D., Mota, J. C. D., Silva, R. S. D., Campos, M. R., \& Schramm, J. M. D. A. (2016). Descrição dos registros repetidos no Sistema de Informação de Agravos de Notificação, Brasil, 2008-2009. Epidemiologia e Serviços de Saúde, 25, 487-498.

Tang, X., Du, R. H., Wang, R., Cao, T. Z., Guan, L. L., Yang, C. Q., Zhu, Q., Hu, M., Li, X., Li, Y., Liang, L., Tong, Z., Sun, B., Peng, P., \& Shi, H. Z. (2020). Comparison of hospitalized patients with ARDS caused by COVID-19 and H1N1. Chest, 158(1), $195-205$.

WHO (2020). WHO Director-General's opening remarks at the media briefing on COVID-19 - 11 March 2020. www.who.int/dg/speeches/detail/who-directorgeneral-s-opening-remarks-at-the-media-briefing-on-covid-19---11-march-2020>.

Wilking, H., Buda, S., Von Der Lippe, E., Altmann, D., Krause, G., Eckmanns, T., \& Haas, W. (2010). Mortality of 2009 pandemic influenza A (H1N1) in Germany. Eurosurveillance, 15(49), 19741.

Zhu, N., Zhang, D., Wang, W., Li, X., Yang, B., Song, J., Zhao, X., Huangm, B., Shi, W., Lu, R., Niu, P., Zhan, F., Ma, X., Wang, D., Xu, W., Wu, G., Gao, G. F., \& Tan, W. (2020). A novel coronavirus from patients with pneumonia in China, 2019. New England journal of medicine, 382 (2). 